\section{ORIGINAL RESEARCH}

\author{
Z. Fan \\ M. Styner \\ J. Muenzer \\ M. Poe \\ M. Escolar
}

\title{
Correlation of Automated Volumetric Analysis of Brain MR Imaging with Cognitive Impairment in a Natural History Study of Mucopolysaccharidosis II
}

\begin{abstract}
BACKGROUND AND PURPOSE: Reliable markers for predicting neurologic outcome in patients with MPS II are lacking. The purpose of this study is to explore whether quantitative volumetric measurements of brain MR imaging can be used to differentiate between MPS II patients with and without cognitive impairment. This MR imaging study is the first in MPS II patients to use automated/semiautomated methods to quantify brain volumes in a longitudinal design.
\end{abstract}

\begin{abstract}
MATERIALS AND METHODS: Sixteen male patients with MPS II in a natural history study had annual brain MR imaging and detailed neurodevelopmental assessment over 2 years. Automated and semiautomated methods were used to determine brain volumes. Linear mixed regression models adjusting for age were used to assess the correlation between the volumetric parameters and cognition.
\end{abstract}

RESULTS: Among the 16 MPS I| patients, 10 (22 MR imaging studies) had cognitive impairment whereas the other 6 (11 MR imaging studies) had normal cognition. A decreased brain tissue/ICV ratio $(-5 \% ; P<.001)$ and an increased lateral ventricle/ICV ratio $(+4 \% ; P=.029)$ were found in patients with cognitive impairment compared with patients with normal cognition. These changes were apparent in patients as young as 7 years of age in addition to older patients.

CONCLUSIONS: Quantitative volumetric measurements of brain MR imaging in MPS II patients can be obtained by using automated and semi-automated segmentation methods. MPS II patients with cognitive impairment have decreased brain tissue volumes, but longer studies with more subjects are required to confirm these results.

ABBREVIATIONS: CNS = central nervous system; ERT = enzyme replacement therapy; ICV = intracranial volume; LV = lateral ventricle; MPS II = mucopolysacchridosis II; PVS = perivascular space; $V P=$ ventriculoperitoneal

$\mathbf{M}$ ucopolysaccharidosis II, or Hunter syndrome, is a rare $\mathrm{X}$-linked recessive lysosomal storage disorder caused by deficiency of iduronate 2-sulfatase, which leads to incomplete degradation and progressive accumulation of glycosaminoglycans in various organs, including the CNS. Clinically, MPS II is characterized by progressive multisystem involvement in the CNS, joints, bones, heart, skin, liver, eyes, and other organs. ${ }^{1}$ The CNS manifestations of MPS II vary widely from severe cognitive impairment to normal cognition; the reason for this heterogeneity is not well understood, but it may be related to the level of residual enzyme activity. ${ }^{2}$ Patients with cognitive impairment

\section{Received October 2, 2009; accepted after revision November 25}

From the Departments of Neurology (Z.F.), Pediatrics (Z.F., J.M.), and Computer Science (M.S.), FPG Child Development Institute (M.P.), and Center for Development and Learning (M.E.), University of North Carolina at Chapel Hill, Chapel Hill, North Carolina.

The MPS II natural history study was sponsored by Shire Human Genetic Therapies, Cambridge, Massachusetts. Data analysis was supported by additional grant funding provided by the University of North Carolina Neurodevelopmental Disorders Research Center (NIH HD 03110) and the Division of Genetics and Metabolism, Department of Pediatrics, University of North Carolina at Chapel Hill.

Please address correspondence to Zheng Fan, MD, Department of Neurology, CB 7025, University of North Carolina at Chapel Hill, 101 Manning Dr, Chapel Hill, NC 27599; e-mail: zhengfan@med.unc.edu

Indicates open access to non-subscribers at www.ajnr.org

DOI 10.3174/ajnr.A2032 typically die in their teenage years, whereas patients with normal cognition can have a nearly normal life span. ${ }^{1,3}$ Intravenous ERT has not altered CNS outcome despite its success in treating some of the non-CNS symptoms in MPS II. ${ }^{4}$ With new therapeutics aimed at treating the CNS manifestation of MPS II on the horizon, there is a need to find reliable markers for predicting neurologic outcome, monitoring the CNS progression, and assessing therapeutic intervention.

Although brain MR imaging has been used to study the CNS abnormalities in MPS II for many years, ${ }^{5-9}$ those studies had limited success in correlating the neuroimaging findings and cognitive function. ${ }^{10-12}$ Vedolin et $\mathrm{l}^{12}$ and Gabrielli et $\mathrm{al}^{10}$ showed that white matter lesions, brain atrophy, and hydrocephalus were more common in patients with cognitive impairment than those with normal cognition; however, Matheus et al did not find a correlation. ${ }^{11}$ These studies were cross-sectional and imaging results were descriptive.

Although recent advances in MR imaging and medical imaging processing have enabled largely automated measurements of cerebral, ventricle, and CSF volumes in many conditions, automating such analysis in MPS II remains challenging. The gross structural abnormality in MPS II makes atlas-based registration by using the automated method difficult. The purpose of this study was to evaluate if automated methods could be used to measure brain tissue volume and if decreased brain volume/ brain atrophy correlates with cognitive impairment. 

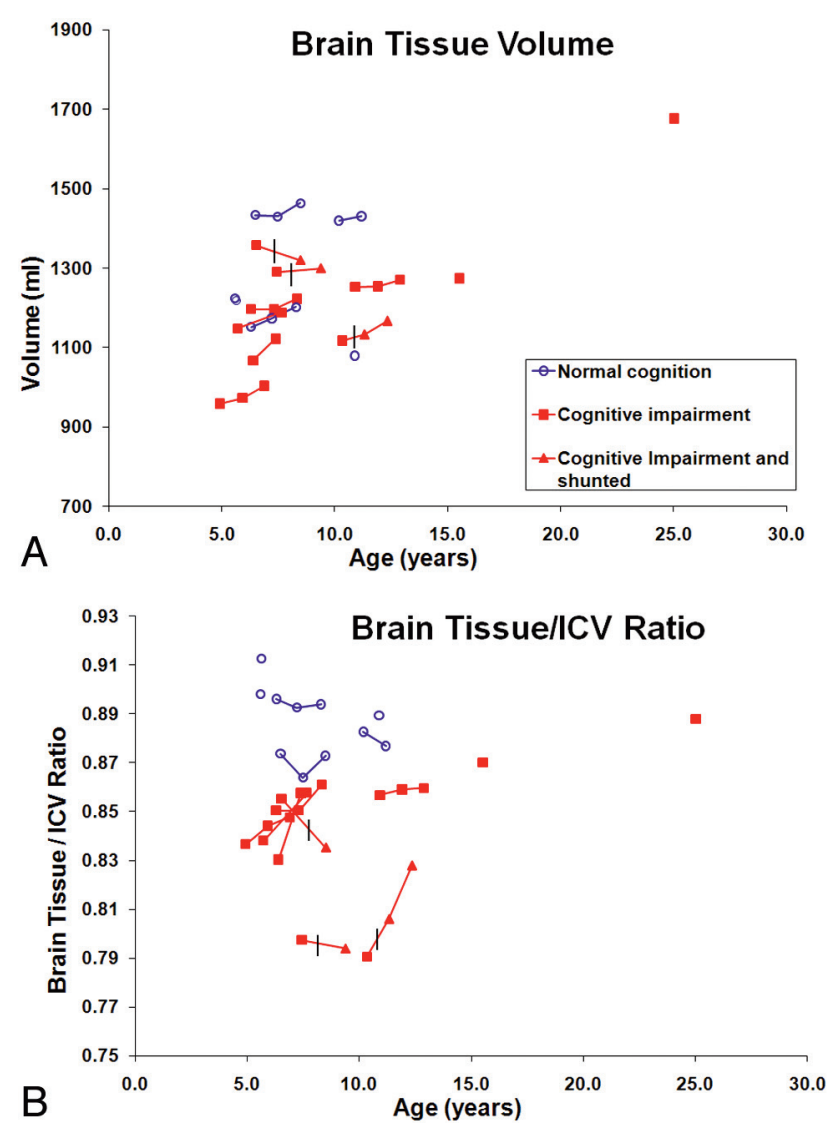

Fig 1. Correlation of brain tissue volume $(A)$ and brain tissue/ICV ratio $(B)$ with cognitive status. The black vertical crossbars indicate the timeline when VP shunt was placed.

\section{Materials and Methods}

\section{Subjects}

Twenty male patients with MPS II were enrolled in a natural history study from 2002 to 2005 at the University of North Carolina; 17 had at least 1 brain MR imaging. These patients had not been treated with ERT or hematopoietic stem cell transplantation. A VP shunt was placed if it was clinically indicated. Some patients discontinued the natural history study when they enrolled in a clinical trial of ERT for MPS II. Each patient presented with typical clinical manifestations of the disorder and had biochemical confirmation of deficient activity of iduronate 2-sulfatase. Multiple sulfatase deficiency was excluded by having a normal activity of at least 1 other sulfatase. Each patient had the brain MR imaging and neurodevelopmental assessment within the same week.

The study was approved by the local institutional review board (02-PED-239). Informed consent was obtained from the patients or their legal representatives.

\section{Neurodevelopmental Assessment}

All patients received age-appropriate standardized neurodevelopmental assessments. ${ }^{13-15}$ The Leiter International Performance ScaleRevised (Leiter) ${ }^{15}$ was used to measure the speech-free intelligence quotient in all patients. Two other assessments were also used as part of the MPS II natural history study, but only the Leiter results were used for classification in this brain imaging study. A subject was classified as having cognitive impairment when the full intelligence quotient was 2 standard deviations below the mean. The Scales of Independent Behavior-Revised ${ }^{13}$ were administered to all parents in a questionnaire form to measure adaptive functioning. The Differential Ability Scales ${ }^{14}$ were used only to supplement areas that were not tested by the Leiter Scale.

\section{Data Acquisition}

MR imaging studies of the brain were obtained on 1.5T Vision magnets (Siemens, Erlangen, Germany). All examinations used the same research protocol that included axial 3-mm T2-weighted (TR/TE, $9900 \mathrm{~ms} / 99 \mathrm{~ms}$; voxel size, $0.86 \times 0.86 \times 3.00$; image dimensions, $256 \times 256 \times 46$; FOV, 193; number of sections, 46) and axial 2-mm continuous T1-weighted MR images (TR/TE, $13.5 \mathrm{~ms} / 7 \mathrm{~ms}$; flip angle, $90^{\circ}$; voxel size, $0.90 \times 0.90 \times 1.97$; image dimensions, $256 \times$ $256 \times 76$; FOV, 173; number of sections, 76) for each subject. Contrast was not used.

\section{Imaging Processing}

All MR imaging analyses were performed at the University of North Carolina Neuro Image Research and Analysis Laboratories. Each dataset (axial T1-weighted and T2-weighted images) was processed by using a fully automated tissue-segmentation method ${ }^{16}$ that generated detailed probability maps of gray matter, white matter, and CSF. An automated, atlas-based brain tissue segmentation algorithm (itkEMS software, version 1.7; http://www.ia.unc.edu/dev/download/itkems/index.htm) was employed with a designated atlas presenting enlarged lateral ventricles. The atlas image was computed locally by the University of North Carolina Neuro Image Research and Analysis Laboratories as the unbiased average image from several scans of subjects presenting with large ventricles and normalappearing white and gray matter. The tissue probability maps were generated by first segmenting the atlas itself with the tissue segmentation method and then smoothing the probabilistic segmentation maps with a Gaussian kernel of $5 \mathrm{~mm}$. The processing procedure includes a bias field correction that adjusts for intensity inhomogeneities. ${ }^{17}$ Furthermore, atlas registration was performed by using a nonrigid, B-spline-based registration. A semi-automated, rater-initiated method was used to segment the LV based on the probabilistic CSF map. ${ }^{18}$ A detailed protocol is accessible at http://www.ia.unc.edu/dev/ tutorials/Documents/UNC-NeuroimagingLab-Manual.pdf. The reproducibility of this method has been validated with intraclass correlation at $0.99 .{ }^{19}$ Total ICV was calculated by summation of gray and white matter and CSF-filled spaces, which include the cerebrum, brain stem, and cerebellum. Total brain tissue was calculated by summation of gray and white matter.

\section{Statistical Analysis}

Descriptive statistics were examined for group differences that could bias the estimates of the differences between the group of patients with cognitive impairment and the group with normal cognition. The cognitively impaired group was older than the cognitively normal group. Thus, we included age (years as a continuous predictor) in the model to adjust its effect. The age at the MR imaging acquisition is marked on the $\mathrm{x}$-axis and the timing of VP shunt placement in relation to MR imaging acquisitions is marked with a black vertical crossbar in Figs 1 and 2. A series of linear mixed-model regressions were fit with the brain volume of interest as the outcome variable (ICV, brain tissue volume, LV volume, brain tissue/ICV ratio, and LV/ICV ratio) and group and age as predictors. Linear mixed models allow for uneven spacing in measurements across time, as well as an unequal number of measurements across subjects. ${ }^{20}$ We also carried out group by age-specific effect analysis in these models. 

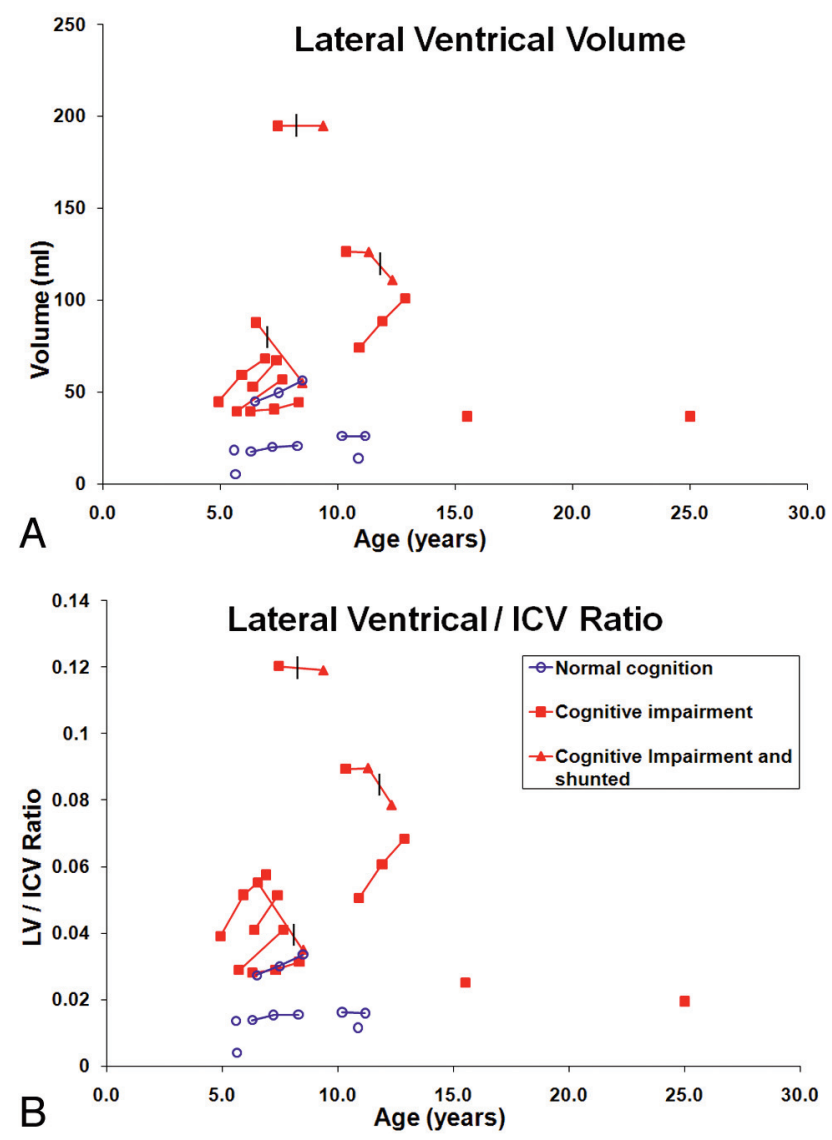

Fig 2. Correlation of $L V$ volume $(A)$ and $L V / I C V$ ratio $(B)$ with cognitive status. The vertical crossbars indicate the timeline when VP shunt was placed.

\section{Results}

Seventeen male MPS II patients were imaged and a total of 34 brain MR imaging studies were obtained over 2 years. One patient with only $1 \mathrm{MR}$ imaging study was excluded due to failed registration that was secondary to grossly abnormal ventricle structure from multiple shunt revisions. A total of 33 brain MR imaging studies on 16 patients were used in further analysis. Six patients had 3 MR imaging studies over 2 years, 5 had 2 MR imaging studies over 1 year, and 5 patients had only $1 \mathrm{MR}$ imaging study. These patients ranged from 4.9 to 25.0 years of age, with an average age of 9.0 years. Although the average age for the group with cognitive impairment (9.9 years) was older than that with normal cognition ( 7.5 years), the difference was not statistically significant $(P=.296)$ (Table).

Brain was segmented into 3 categories: gray matter, white matter, and CSF fluid (examples are shown in Fig 3). We observed that the segmentation between gray matter and white matter was compromised as a result of low gray-white contrast on brain MR imaging in some MPS II patients, and thus no separate analysis for white and gray matter was performed. The segmentation of enlarged PVSs was variable, largely based on their size: larger ones (larger than $3 \times 3 \times 3 \mathrm{~mm}^{3}$ ) were consistently segmented as CSF fluid, whereas the smaller ones (smaller than $3 \times 3 \times 3 \mathrm{~mm}^{3}$ ) were either segmented as CSF or white matter. Thirty-three MR imaging studies were dichotomized, which included patients with cognitive impairment (22 MR imaging studies in 10 patients) and patients with normal cognition (11 MR imaging studies in 6 patients) (Table). No patients changed cognitive classification during this study. We asked if cognitive status is correlated with the following volumetric measurements: ICV, brain tissue volume, LV volume, brain tissue/ICV ratio, and LV/ICV ratio with a model adjusted for age (Table). We found that the brain tissue/ICV ratio is significantly decreased in patients with cognitive impairment compared with those with normal cognition $(-5 \%$; $P<.001)$; however, the brain tissue volume itself is not significantly different between the 2 groups $(P=.25)$ (Fig 1). Furthermore, both $\mathrm{LV} / \mathrm{ICV}$ ratio $(+4 \% ; P=.029)$ and $\mathrm{LV}$ volume $(+52 \mathrm{~mL} ; P=.046)$ were significantly larger in patients with cognitive impairment than in patients with normal cognition (Fig 2). One patient with normal cognition had a LV/ICV ratio that overlapped with those of patients with cognitive impairment (Fig $2 B$ ), but the brain tissue/ICV ratio distinguished this patient from the patients with cognitive impairment (Fig $1 B)$. It was also noted that 2 of the 3 shunted patients had the lowest brain tissue/ICV ratio (Fig $1 B$ ). These volumetric differences were apparent in our youngest patients $(9$ studies in patients $<7$ years of age including the youngest at 4.9 years of age) in addition to the older patients.

Finally, we carried out the same analysis by excluding patients/studies with VP shunt (4 studies, all in patients with cognitive impairment). The volumetric changes were similar and remained statistically significant $(P<.001$ for brain tissue/ICV with cognitive status and $P=.033$ for LV/ICV with cognitive status). Group by age-specific effects for both the cognitively impaired and cognitively normal were calculated for brain tissue/ICV in all studies (33 total), LV/ICV in all studies (33 total), brain tissue/ICV in nonshunted studies (29 total), and LV/ICV in nonshunted studies (29 total). Agespecific effects (difference in group-specific slopes) were not statistically significant except for the brain tissue/ICV ratio in the cognitively impaired group (nonshunted) $(P<.001)$.

Descriptively, brain MR imaging findings in MPS II patients appeared to vary widely, ranging from near normal appearing brain to grossly abnormal brain. Four patients had markedly enlarged ventricles and 2 of these had quite asymmetric lateral ventricles. All 16 patients had some degree of enlarged PVSs, though the severity varied widely. The most commonly enlarged PVSs in our study were in periventricular and subcortical white matter, followed by corpus callosum, thalamus, and occasionally in basal ganglia, but none in brain stem. Major events occurred during the study: 3 patients received VP shunt placement and 1 patient developed a unilateral subdural hematoma that stabilized without intervention.

\section{Discussion}

Our results demonstrate that quantitative volumetric measurements of brain MR imaging in MPS II patients can be obtained by using automated/semi-automated segmentation methods and that MPS II patients with cognitive impairment have decreased brain tissue volumes. It is typically assumed that decreased brain volume reflects brain atrophy, which results in or at least contributes to cognitive impairment in MPS II patients. However, longer duration studies starting with younger patients are needed to conclude that the reduced brain volume we observed may represent an acquired process (atrophy), not abnormal brain development. 


\begin{tabular}{|c|c|c|c|c|}
\hline \multicolumn{5}{|c|}{ Volumetric analysis of brain MR Imaging in MPS II Patients } \\
\hline Variables & $\begin{array}{c}\text { All Patients MR } \\
\text { Imaging Studies } \\
(N=33) \text {, Mean (SD) }\end{array}$ & $\begin{array}{l}\text { Patients with Cognitive } \\
\text { Impairment MR Imaging Studies } \\
(N=22) \text {, Mean (SD) }\end{array}$ & $\begin{array}{c}\text { Patients with Normal } \\
\text { Cognition MR Imaging Studies } \\
(N=11) \text {, Mean (SD) }\end{array}$ & $P$ Value $^{\mathrm{a}}$ \\
\hline Age (years) & $9.0(5.0)$ & $9.9(6.2)$ & $7.5(2.37)$ & .296 \\
\hline ICV (mL) & $1443(189)$ & $1464(201)$ & $1407(176)$ & .96 \\
\hline Brain tissue $(\mathrm{mL})$ & $1242(173)$ & $1234(195)$ & $1254(143)$ & .25 \\
\hline $\mathrm{LV}(\mathrm{mL})$ & $54(48)$ & $73(52)$ & $21(13)$ & $.046^{b}$ \\
\hline Brain tissue/ICV & $0.86(0.012)$ & $0.84(0.03)$ & $0.89(0.01)$ & $<.001^{b}$ \\
\hline LV/ICV & $0.04(0.015)$ & $0.05(0.03)$ & $0.01(0.01)$ & $.029^{b}$ \\
\hline
\end{tabular}

a $P$ values were comparisons between patients with cognitive impairment and those with normal cognition.

b $P$ values reached statistical significance.
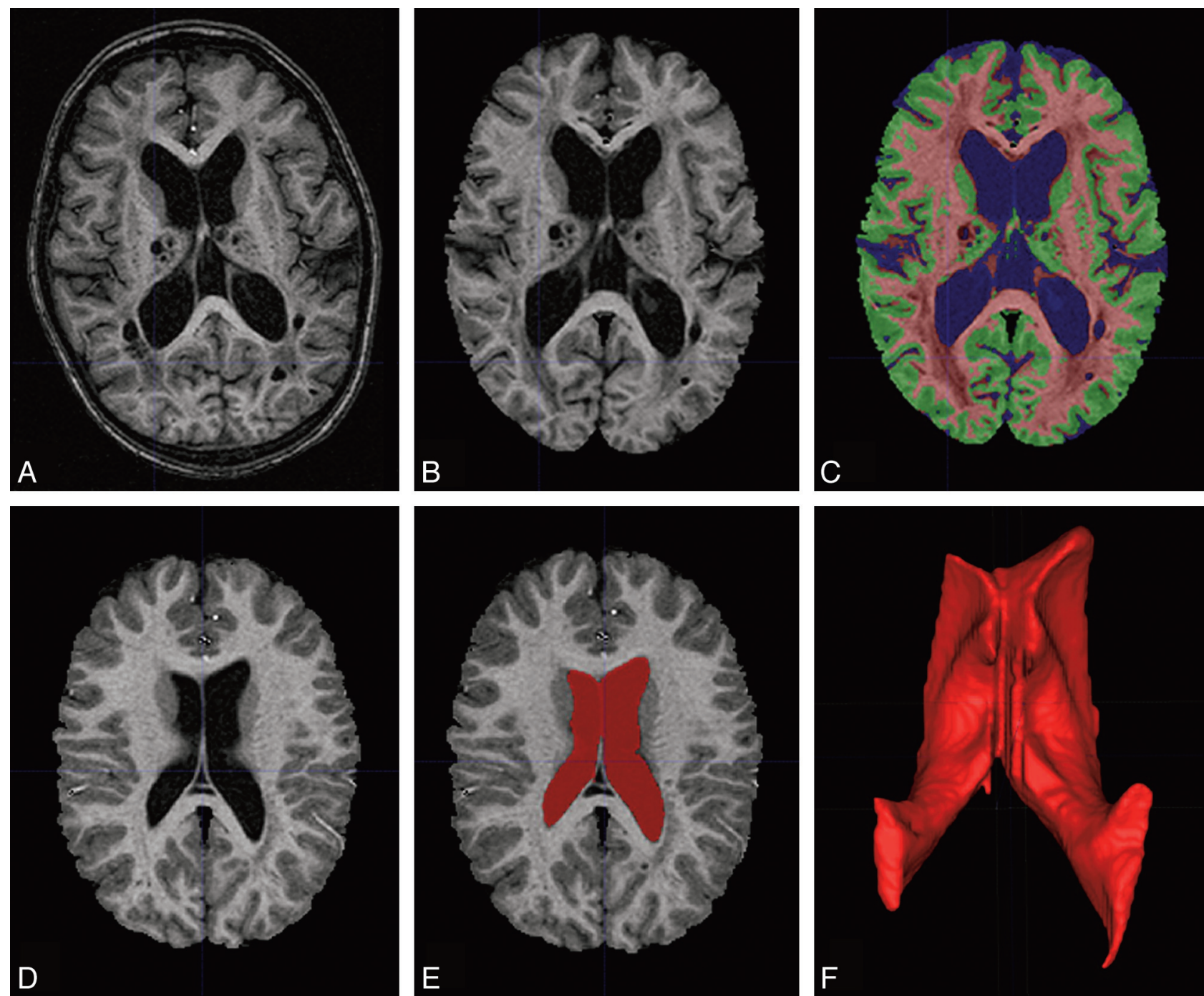

Fig 3. Examples of the automated segmentation of brain and semi-automated segmentation of lateral ventricles. $A$, Original axial $\mathrm{T} 1$ image; $B$, aligned and registered $\mathrm{T} 1 \mathrm{image}, C$, segmented image, where green voxels represent gray matter, pink voxels represent white matter, and dark blue voxels represent CSF; $D$, registered T1 axial image; $E$, $2 \mathrm{D}$ view of the segmented lateral ventricles in red; $F, 3 D$ view of the lateral ventricles (enlarged).

Our study shows that the ratios (brain tissue volume and LV volume expressed by fraction of ICV), rather than the absolute volumes (brain tissue and LV), are better correlated with cognitive status. We think that the ratios (tissue/ICV and LV/ICV) are more indicative of progressive brain atrophy, because they take the baseline individual brain volume differences into account. We suggest that brain tissue/ICV ratio may be a useful marker for global brain atrophy and the LV/ICV ratio for subcortical atrophy.
Our results are in agreement with those from Vedolin et $\mathrm{al}^{12}$ who showed that brain atrophy was more common in MPS II patients with cognitive impairment than those without. The MR imaging study by Matheus et al ${ }^{11}$ only included cognitively intact MPS II patients. It is not surprising that no association between neuroimaging findings and clinical manifestations was found. Both were descriptive studies. We think that our study is the first longitudinal study in MPS II patients by using automated/semiautomated methods to quantify brain volumes. 
Our results also suggest that brain MR imaging volumetric changes between patients with and without cognitive impairment may be apparent in children as young as 7 years of age (9 studies in patients from 4.9 to 7 years of age; Figs 1 and 2) in addition to older patients. This result suggests that these quantitative volumetric markers may be useful for identifying early changes in disease progression. Documenting such changes may allow us to distinguish those MPS II patients who may develop cognitive decline from those who may not. Consequently, early intervention during this critical window may be possible.

Differences between group-specific slopes in a mixed model allow us to evaluate the average trajectories for each group (cognitively impaired and cognitively normal) that are estimated from longitudinal data. The group-by-age specific effect was found significant only for the brain tissue/ICV ratio in the cognitively impaired nonshunted group. The group with normal cognition had a higher brain tissue/ICV ratio than the cognitively impaired group and it showed no significant change with age. The cognitively impaired group had a lower brain tissue/ICV ratio than the cognitively normal group but the ratio appears to increase with age in the nonshunted population (Fig $1 B$ ). Statistically, we noted that the 3 shunted patients contributed to the large variation in the cognitively impaired group, thus making the group-by-age specific effect nonsignificant. Only when the $4 \mathrm{MR}$ imaging studies (3 patients) obtained after the patients were shunted were removed from the analysis did the age effect become statistically significant. Biologically, we do not have a good explanation for an increased brain tissue/ICV ratio with age in only the cognitively impaired nonshunted MPS II patients. We also point out that the VP shunt may be a surrogate marker for the most severely affected MPS II patients. Removing the shunted MR imaging studies may skew the severity of the imaging phenotype in the cognitive impaired group.

\section{Conclusions}

Our data demonstrate that quantitative volumetric measurements of brain MR imaging in MPS II patients can be obtained by using automated or semi-automated segmentation methods. MPS II patients with cognitive impairment have decreased brain tissue volumes. Our study suggests that the total brain tissue volume and LV volume expressed by fraction of ICV may be used as markers for cognitive impairment and that these changes may be apparent in MPS II patients as young as 7 years of age as well as in older patients. Larger and longer prospective longitudinal MR imaging volumetric studies in young MPS II patients starting before they develop cognitive impairment are needed to confirm our results.

\section{References}

1. Neufeld EF, Muenzer J. The mucopolysaccharidoses. In: Scriver CR, Beadeut AL, Sly WS, eds. The Metabolic and Molecular Bases of Inherited Disease. New York: McGraw-Hill; 2001:3421-52

2. Vedolin L, Schwartz IV, Komlos M, et al. Brain MRI in mucopolysaccharidosis: effect of aging and correlation with biochemical findings. Neurology 2007; 69:917-24

3. Meikle PJ, Hopwood JJ, Clague AE, et al. Prevalence of lysosomal storage disorders. JAMA 1999;281:249-54

4. Muenzer J, Gucsavas-Calikoglu M, McCandless SE, et al. A phase I/II clinical trial of enzyme replacement therapy in mucopolysaccharidosis II (Hunter syndrome). Mol Genet Metab 2007;90:329-37

5. Lee C, Dineen TE, Brack M, et al. The mucopolysaccharidoses: characterization by cranial MR imaging. AJNR Am J Neuroradiol 1993;14:1285-92

6. Murata R, Nakajima S, Tanaka A, et al. MR imaging of the brain in patients with mucopolysaccharidosis. AJNR Am J Neuroradiol 1989;10:1165-70

7. Parsons VJ, Hughes DG, Wraith JE. Magnetic resonance imaging of the brain, neck and cervical spine in mild Hunter's syndrome (mucopolysaccharidoses type II). Clin Radiol 1996;51:719-23

8. Seto T, Kono K, Morimoto K, et al. Brain magnetic resonance imaging in 23 patients with mucopolysaccharidoses and the effect of bone marrow transplantation. Ann Neurol 2001;50:79-92

9. Shimoda-Matsubayashi S, Kuru Y, Sumie H, et al. MRI findings in the mild type of mucopolysaccharidosis II (Hunter's syndrome). Neuroradiology 1990;32: 328-30

10. Gabrielli O, Polonara G, Regnicolo L, et al. Correlation between cerebral MRI abnormalities and mental retardation in patients with mucopolysaccharidoses. Am J Med Genet A 2004;125A:224-31

11. Matheus MG, Castillo M, Smith JK, et al. Brain MRI findings in patients with mucopolysaccharidosis types I and II and mild clinical presentation. Neuroradiology 2004;46:666-72

12. Vedolin L, Schwartz IV, Komlos M, et al. Correlation of MR imaging and MR spectroscopy findings with cognitive impairment in mucopolysaccharidosis II. AJNR Am J Neuroradiol 2007;28:1029-33

13. Bruininks R, Woodcock W, Weatherman R, et al. The Scales of Independent Behavior-Revised. New York: Riverside Publishing; 1996

14. Elliott CD. Differential Ability Scales, 2nd ed. New York: Harcourt Brace Jovanovich; 2006

15. Roid G, Miller L. Leiter International Performance Scale-Revised. Wood Dale, Illinois: Stoelting; 1997

16. Van Leemput K, Maes F, Vandermeulen D, et al. Automated model-based tissue classification of MR images of the brain. IEEE Trans Med Imaging 1999; 18:897-908

17. Styner M, Brechbuhler C, Szekely G, et al. Parametric estimate of intensity inhomogeneities applied to MRI. IEEE Trans Med Imaging 2000;19: 153-65

18. Yushkevich PA, Piven J, Hazlett HC, et al. User-guided 3D active contour segmentation of anatomical structures: significantly improved efficiency and reliability. Neuroimage 2006;31:1116-28

19. Styner M, Charles C, Park J, et al. Multisite validation of image analysis methods: assessing intra- and intersite variability. Proc SPIE Med Imaging 2002; $4684: 278-86$

20. Singer JD, Willett JB. Applied Longitudinal Data Analysis: Modeling Change and Event Occurrence. New York: Oxford University Press; 2003 